\title{
Neurocognitive function in HIV-infected persons with asymptomatic cryptococcal antigenemia: a comparison of three prospective cohorts
}

Martha P. Montgomery ${ }^{1 \dagger}$, Noeline Nakasujja ${ }^{2,3,4,8^{*}+}$, Bozena M. Morawski ${ }^{1,5}$, Radha Rajasingham ${ }^{1}$, Joshua Rhein ${ }^{1,2}$, Elizabeth Nalintya ${ }^{2}$, Darlisha A. Williams ${ }^{1,2}$, Kathy Huppler Hullsiek ${ }^{5}$, Agnes Kiragga $^{2}$, Melissa A. Rolfes ${ }^{5}$, Renee Donahue Carlson ${ }^{1}$, Nathan C. Bahr ${ }^{1}$, Kate E. Birkenkamp ${ }^{1}$, Yukari C. Manabe ${ }^{2,6}$, Paul R. Bohjanen ${ }^{1,2}$, Jonathan E. Kaplan', Andrew Kambugu ${ }^{1,2,3}$, David B. Meya ${ }^{1,2,3+}$, David R. Boulware ${ }^{1,5^{*}+}$

and on behalf of the COAT and ORCAS Trial Teams

\begin{abstract}
Background: HIV-infected persons with detectable cryptococcal antigen (CrAg) in blood have increased morbidity and mortality compared with HIV-infected persons who are CrAg-negative. This study examined neurocognitive function among persons with asymptomatic cryptococcal antigenemia.

Methods: Participants from three prospective HIV cohorts underwent neurocognitive testing at the time of antiretroviral therapy (ART) initiation. Cohorts included persons with cryptococcal meningitis ( $N=90)$, asymptomatic $\mathrm{CrAg}+(N=87)$, and HIV-infected persons without central nervous system infection $(N=125)$. Z-scores for each neurocognitive test were calculated relative to an HIV-negative Ugandan population with a composite quantitative neurocognitive performance Z-score (QNPZ-8) created from eight tested domains. Neurocognitive function was measured pre-ART for all three cohorts and additionally after 4 weeks of ART (and 6 weeks of pre-emptive fluconazole) treatment among asymptomatic CrAg + participants.
\end{abstract}

Results: Cryptococcal meningitis and asymptomatic CrAg + participants had lower median CD4 counts (17 and 26 cells/ $\mu \mathrm{L}$, respectively) than the HIV-infected control cohort (233 cells/ $\mu \mathrm{L}$ ) as well as lower Karnofsky performance status (60 and 70 vs. 90, respectively). The composite QNPZ-8 for asymptomatic CrAg + (-1.80 Z-score) fell between the cryptococcal meningitis cohort $(-2.22$ Z-score, $P=0.02)$ and HIV-infected controls $(-1.36, P=0.003)$. After four weeks of ART and six weeks of fluconazole, the asymptomatic CrAg + cohort neurocognitive performance improved (-1.0 Z-score, $P<0.001)$.

Conclusion: Significant deficits in neurocognitive function were identified in asymptomatic CrAg + persons with advanced HIV/AIDS even without signs or sequelae of meningitis. Neurocognitive function in this group improves over time after initiation of pre-emptive fluconazole treatment and ART, but short term adherence support may be necessary.

Keywords: AIDS dementia complex, Neurocognitive disorders, Cryptococcal meningitis, HIV, Neuropsychological tests, Cryptococcus

\footnotetext{
* Correspondence: drnoeline@yahoo.com; boulw001@umn.edu

${ }^{\dagger}$ Equal contributors

${ }^{2}$ Infectious Disease Institute, Makerere University, Kampala, Uganda

1 Division of Infectious Diseases and International Medicine, Department of

Medicine, University of Minnesota, 3-222 MTRF, 2001 6th St SE, Minneapolis,

MN 55455, USA

Full list of author information is available at the end of the article
} 


\section{Background}

Cryptococcal meningitis is one of the leading causes of death from HIV/AIDS in sub-Saharan Africa [1, 2] causing 15-20\% of AIDS-related mortality [3]. In the United States, cryptococcal meningitis is more common than all causes of bacterial meningitis combined (1.1 vs. 0.73 per 100,000 people, respectively) [4, 5]. Yet cryptococcal disease is potentially preventable as cryptococcal antigen ( $\mathrm{CrAg})$ is detectable in the peripheral blood weeks to months prior to the onset of symptomatic meningitis. Although many of these $\mathrm{CrAg}+$ persons exhibit no clinical signs or symptoms despite the presence of early disseminated infection, CrAg + persons have increased risk of developing meningitis or death compared to HIVinfected persons without cryptococcal antigenemia [6-9].

The World Health Organization (WHO) and U.S. HIV guidelines recommend routine $\mathrm{CrAg}$ screening for HIVinfected persons with $<100 \mathrm{CD} 4 \mathrm{~T}$ cells $/ \mu \mathrm{L}$ who are not receiving effective antiretroviral therapy (ART), along with pre-emptive fluconazole therapy for those screening $\mathrm{CrAg}+[10,11]$. Despite these recommendations, many gaps remain in understanding the morbidity and clinical course of asymptomatic CrAg + persons.

Persons living with HIV, regardless of their CrAg serostatus, have increased risk of neurocognitive deficits, presumably through the direct toxic effect of the HIV virus itself and consequent inflammation. The wide array of observed disorders is referred to collectively as HIV-associated neurocognitive disorder (HAND), which ranges from mild cognitive impairment to the more extreme HIV-associated dementia [12]. One prevalence study estimated that $31 \%$ of people living with HIV in Uganda have HIV dementia and that people with older age and lower CD4 counts are at higher risk [13].

Opportunistic infections of the central nervous system (CNS) create potential for further damage, and HIVinfected persons with cryptococcal meningitis, specifically, are known to have increased neurocognitive deficits compared with their HIV-infected, CrAg-negative peers [14, 15]. It is not known whether cryptococcal antigenemia increases the risk of neurocognitive dysfunction in asymptomatic $\mathrm{CrAg}+$ persons, which may have an implication for additional needed support for general HIV care. The purpose of this study was to 1 ) investigate neurocognitive changes among HIV-infected persons with asymptomatic cryptococcal antigenemia, and 2) compare neurocognitive performance of these asymptomatic $\mathrm{CrAg}+$ persons to HIV-infected persons with cryptococcal meningitis and those without CNS infection.

\section{Methods}

This study utilized three cohorts of HIV-infected ARTnaïve persons enrolled in prospective clinical trials and cohort studies in Kampala, Uganda. All participants provided written, informed consent prior to enrollment. Studies were approved by institutional review boards in Uganda and Minnesota.

\section{Study populations}

The first population consisted of HIV-infected persons hospitalized with cryptococcal meningitis who survived to the one-month follow-up visit. These participants were prospectively enrolled into a clinical trial to determine optimal timing of ART after the first episode of cryptococcal meningitis (ClinicalTrials.gov: NCT01075152) [14, 16]. Cryptococcal meningitis was diagnosed based on cerebrospinal fluid (CSF) culture and/or CSF CrAg lateral flow assay (Immy, Norman, Oklahoma). Participants diagnosed with cryptococcal meningitis were treated with combined amphotericin B deoxycholate $(0.7-1.0 \mathrm{mg} / \mathrm{kg} /$ day) plus fluconazole $800 \mathrm{mg} /$ day in a two-week induction therapy regimen, followed by enhanced consolidation therapy of fluconazole of $800 \mathrm{mg} /$ day for $\sim 3$ weeks, then $400 \mathrm{mg} /$ day for 8 weeks, and then secondary prophylaxis with fluconazole $200 \mathrm{mg} /$ day. ART was initiated at either 12 weeks following cryptococcal meningitis diagnosis or 5 weeks after diagnosis, based on the randomized trial arm in the main clinical trial. Baseline neurocognitive testing was performed at approximately 1 month after meningitis diagnosis. We have previously shown that there was no difference in baseline or longitudinal neurocognitive function based on early vs. deferred ART. Additional study details have been published previously $[14,16]$.

The second study population included HIV-infected, ART-naïve persons who were $\mathrm{CrAg}+$ in plasma after labbased reflexive screening among persons with CD4 < 100 cells $/ \mu \mathrm{L}$, but without symptoms of cryptococcal meningitis (ClinicalTrials.gov: NCT01535469). Participants were enrolled prospectively and followed for 6 months. All asymptomatic $\mathrm{CrAg}+$ persons received pre-emptive fluconazole therapy at $400 \mathrm{mg}$ twice daily for 2 weeks, followed by $400 \mathrm{mg}$ daily for the subsequent 8 weeks. ART was started 2 weeks after initiation of fluconazole. Neurocognitive testing was performed at the ART initiation visit and then again 4 weeks after ART initiation.

Third, to account for the potential effect of HAND, we included a population of HIV-infected persons enrolled in an outpatient clinic setting [17]. HIV-infected adults were screened consecutively for enrollment, and those with clinical signs or symptoms of meningitis or with a history of CNS infection were excluded. Additional exclusion criteria included active psychiatric disease, alcoholism, or physical deficits that would interfere with neurocognitive testing. In this cohort, neurocognitive testing was performed once prior to ART initiation. 


\section{Neurocognitive testing}

Participants in all three cohorts underwent outpatient neurocognitive testing with a neuropsychological battery of tests. Testing for all three cohorts and for the retesting of the asymptomatic $\mathrm{CrAg}+$ cohort was performed by the same team using the same study forms and was conducted in participant's native language. The assessments explored the following neurocognitive domains: Attention span: Digit Span Forward and Backward [18] and Color Trails 1 [19]. Processing speed: Digit Symbol test [18] and Color Trails 1 [19]. Executive function: Color Trails 2 [19]. Verbal learning: WHO-UCLA (University of California, Los Angeles) Auditory Verbal Learning Test-Revised [20]. Language fluency: Semantic Verbal Fluency [21]. Motor speed: Finger Tapping [22]. Fine motor skills: Grooved Pegboard [23]. Additional file 1 provides a description of each of these tests. Each test score was compared with a standard reference population of HIV-negative Ugandans matched on age and education to produce an age and education adjusted Z-score [24, 25]. Negative Z-scores indicate a worse performance compared with the reference population. A composite score using all eight tests was calculated to create a Quantitative Neurological Performance Z-score (QNPZ-8 score) [26].

In addition to neurocognitive testing, all three populations were evaluated for functional status with the Karnofsky performance status scale [27]. Participants were screened for dementia using the International HIV Dementia Scale (IHDS) [24]. Total scores range from 0 to 12 with a score $\leq 10$ being $80 \%$ sensitive and $55 \%$ specific for HIV dementia. Depression was assessed using the Center for Epidemiologic Studies Depression (CES-D) Scale [28], which ranges from 0 to 60 . Higher scores correspond with increased chance of depression, and a score of 16 or greater has good sensitivity and specificity for identifying clinical depression [29, 30]. All enrolled participants who completed baseline neurocognitive testing were included in the analyses.

\section{Data analysis}

Data were analyzed using STATA/IC version 9.2 (StataCorp, College Station, TX, USA) and are available online in a supplemental file (Additional file 2). Participants who initiated the neurocognitive tests but were too sick to complete all tests attempted as many neurocognitive tests as possible and skipped the remainder. For each skipped/uncompleted test, participants were assigned a value of -2 standard deviations below the cohort mean for that test, as has been done previously [14]. Comparisons of variables among populations were made using unpaired Student's t-tests, Mann-Whitney U tests, and chi-square tests as appropriate. Linear regression was used to identify factors associated with QNPZ-8 score. Comparisons of neurocognitive performance over time were made using paired t-tests of Z-scores.

\section{Results}

Participants with asymptomatic cryptococcemia were slightly younger in age (median 31 years, interquartile range (IQR): 27-39 years) compared with participants with cryptococcal meningitis (36 years, IQR: $30-40$ ) and HIVinfected controls (37, IQR: 32-41) (Table 1). There were significantly more women in the HIV control population (68\% women) relative to patients with Cryptococcus infection. Median education level ranged from 7 to 8 years in the three groups. CD4 count, Karnofsky score, CES-D scale, and IHDS were variable among the three groups. Participants with cryptococcal meningitis had the lowest median CD4 count (17 cells/ $\mu \mathrm{L}, \mathrm{IQR}: 7-79$ ), the lowest median Karnofsky score (60, IQR: 50-70), the highest proportion of participants with IHDS $<=10(92 \%)$, and the highest median scores for depression (CES-D: 23, IQR: 16-30). At the other end of the spectrum, the HIV-infected controls had the lowest proportion with IHDS $<=10(66 \%)$, the lowest depression scale scores (CES-D: 9, IQR: 5-17), highest Karnofsky performance score (90, IQR: 90-90), and significantly higher median CD4 counts (233 cells/ $\mu \mathrm{L}, \mathrm{IQR}$ : 183297) compared with the other two study populations. Overall, asymptomatic CrAg + participants fell in between the two populations and were notably more similar to cryptococcal meningitis survivors with regard to $\mathrm{CD} 4$ count (median 26 cells/ $\mu \mathrm{L}$, IQR: 9-59).

Individual pre-ART neurocognitive test Z-scores for each population are displayed in Fig. 1 (see Additional file 3). Asymptomatic $\mathrm{CrAg}+$ persons performed significantly worse than HIV-infected controls in every domain except for the Color Trails 1 and Grooved Pegboard tests. In addition, asymptomatic CrAg + persons did not significantly differ from cryptococcal meningitis survivors in any measure except for the Grooved Pegboard and Color Trails 1 tests (i.e., domains of speed of information processing, attention, fine motor). With respect to the composite QNPZ8 score, the three study populations were significantly different from each other, with cryptococcal meningitis survivors performing the worst (QNPZ-8 $=-2.22$ vs -1.80 among asymptomatic $\mathrm{CrAg}+, P=0.02)$ and HIV-infected controls performing the best $(\mathrm{QNPZ}-8=-1.36, P=0.003$ compared with asymptomatic $\mathrm{CrAg}+$ ).

There were slight demographic and other variable differences across the three cohort groups, thus a multiple linear regression analysis was performed to assess for independent risk factors associated with decreased neurocognitive performance (Table 2). In an adjusted multivariable model, the QNPZ-8 scores for HIV-infected controls remained significantly higher than QNPZ-8 scores for asymptomatic CrAg + persons (QNPZ-8: +0.42 , 95\% confidence interval (CI): 0.03 to 0.81 ). The asymptomatic $\mathrm{CrAg}+$ persons did not statistically differ from persons with recent cryptococcal meningitis (QNPZ-8: -0.26, 95\% CI: -0.59 to 0.07 ). For each 10 year-increase in age, participants' QNPZ-8 decreased by 
Table 1 Background and clinical characteristics of three study populations

\begin{tabular}{|c|c|c|c|c|c|c|c|c|c|c|c|}
\hline & Cohor & & & Coho & & & Coho & rt $C$ & & & \\
\hline & Crypt & occal mer & tis $(N=90)$ & Asym & tomatic $\mathrm{Cl}$ & $+(N=87)$ & HIV+ & controls ( & $N=125)$ & & \\
\hline & $\mathrm{N}$ & $\begin{array}{l}\% \text { or } \\
\text { Median }\end{array}$ & (IQR) & $\mathrm{N}$ & $\begin{array}{l}\% \text { or } \\
\text { Median }\end{array}$ & (IQR) & $\mathrm{N}$ & $\begin{array}{l}\% \text { or } \\
\text { Median }\end{array}$ & (IQR) & $\begin{array}{l}A \text { vs B } \\
P \text {-value }\end{array}$ & $\begin{array}{l}B \text { vs } C \\
P \text {-value }\end{array}$ \\
\hline Age (years) & 90 & 36 & $(30-40)$ & 87 & 31 & $(27-39)$ & 125 & 37 & $(32-41)$ & 0.02 & $<0.001$ \\
\hline Sex & 90 & & & 87 & & & 125 & & & & \\
\hline Male & & $60 \%$ & & & $51 \%$ & & & $32 \%$ & & & \\
\hline Female & & $40 \%$ & & & $49 \%$ & & & $68 \%$ & & 0.21 & 0.007 \\
\hline Education level (years) & 90 & 8 & $(4-11)$ & 87 & 7 & $(3-11)$ & 125 & 8 & $(6-11)$ & 0.15 & 0.05 \\
\hline$<7$ years & & $36 \%$ & & & $45 \%$ & & & $27 \%$ & & & \\
\hline 7 to 12 years & & $51 \%$ & & & $44 \%$ & & & $62 \%$ & & & \\
\hline$>12$ years & & $13 \%$ & & & $11 \%$ & & & $10 \%$ & & 0.45 & 0.02 \\
\hline CD4 count (cells/uL) & 79 & 17 & $(7-79)$ & 86 & 26 & $(9-59)$ & 125 & 233 & $(183-297)$ & 0.93 & $<0.001$ \\
\hline Karnofsky score & 90 & 60 & (50-70) & 87 & 70 & $(60-80)$ & 125 & 90 & $(90-90)$ & $<0.001$ & $<0.001$ \\
\hline 40 & & $1 \%$ & & & $0 \%$ & & & $0 \%$ & & & \\
\hline 50 & & $28 \%$ & & & $11 \%$ & & & $0 \%$ & & & \\
\hline 60 & & $36 \%$ & & & $20 \%$ & & & $1 \%$ & & & \\
\hline 70 & & $29 \%$ & & & $26 \%$ & & & $0 \%$ & & & \\
\hline 80 & & $3 \%$ & & & $29 \%$ & & & $2 \%$ & & & \\
\hline 90 & & $3 \%$ & & & $14 \%$ & & & $97 \%$ & & & \\
\hline International HIV dementia scale & 88 & & & 87 & & & 125 & & & & \\
\hline$<=10$ & & $92 \%$ & & & $75 \%$ & & & $66 \%$ & & & \\
\hline$>10$ & & $8 \%$ & & & $25 \%$ & & & $34 \%$ & & 0.002 & 0.20 \\
\hline CES depression scale & 88 & 23 & $(16-30)$ & 87 & 18 & $(10-26)$ & 125 & 9 & $(5-17)$ & 0.007 & $<0.001$ \\
\hline$<16$ & & $25 \%$ & & & $41 \%$ & & & $70 \%$ & & & \\
\hline$>=16$ & & $75 \%$ & & & $59 \%$ & & & $30 \%$ & & 0.02 & $<0.001$ \\
\hline
\end{tabular}

Twenty participants from the cryptococcal meningitis cohort and 28 asymptomatic CrAg + participants were excluded due to insufficient data to calculate a QNPZ-8 score. P-values were calculated from student's T-test (comparing means), Mann-Whitney U test, and chi-square test as appropriate

-0.31 (95\% CI: -0.48 to -0.15 ), and participants with CES$\mathrm{D}$ depression scores $\geq 16$ had a mean difference of -0.56 QNPZ-8 score (95\% CI: -0.82 to -0.30). In the multivariable model, CD4 count was not significantly associated with neurocognitive function after adjusting for study cohort (QNPZ-8 $=0.02$ per $100 \mathrm{CD} 4$ cells $/ \mu \mathrm{L}, 95 \% \mathrm{CI}$ : -0.10 to 0.14; $P=0.79$ ). Baseline pre-ART plasma $\mathrm{CrAg}$ titer was also not associated with QNPZ-8 score among the $\mathrm{CrAg}+$ and cryptococcal meningitis cohorts $(P=0.41$, data not shown).

Lastly, we examined neurocognitive performance among asymptomatic $\mathrm{CrAg}+$ persons over time, comparing the pre-ART time point after 2 weeks of fluconazole and a time after 6 weeks of fluconazole plus 4 weeks of ART (Table 3). All measures showed significant improvement after 4 weeks of ART, except for Digit Span Forward, which tests the domains of working memory and attention. The overall QNPZ-8 score improved in the first 4 weeks of ART by +0.7 standard deviations of the population $(P<0.001)$. The baseline characteristics for asymptomatic CrAg + persons also improved significantly over 4 weeks. Average CD4 count increased from 32 cells $/ \mu \mathrm{L}$ to 80 cells $/ \mu \mathrm{L}(P<0.001)$ and average depression scores decreased from 19 to $16(P=0.04)$. Mean Karnofsky performance scores modestly increased from 72 to 75 out of a 100 -point scale $(P=0.04)$, indicating that participants on average were able to care for themselves but unable to carry on normal activity or to do active work.

\section{Discussion}

In this analysis of HIV-infected persons, we found that asymptomatic cryptococcal antigenemia is associated with more neurocognitive deficits compared with HIV-infected persons without a history of CNS infection. On several measures of neurocognitive function, HIV-infected $\mathrm{CrAg}+$ persons did not differ significantly from survivors of cryptococcal meningitis who were tested at 1 month after meningitis diagnosis [14]. Secondly, longitudinal assessment of asymptomatic CrAg + persons found that they had significant improvement in neurocognitive function on most neurocognitive measures after the first 4 weeks of ART. These findings have important practical implications. 


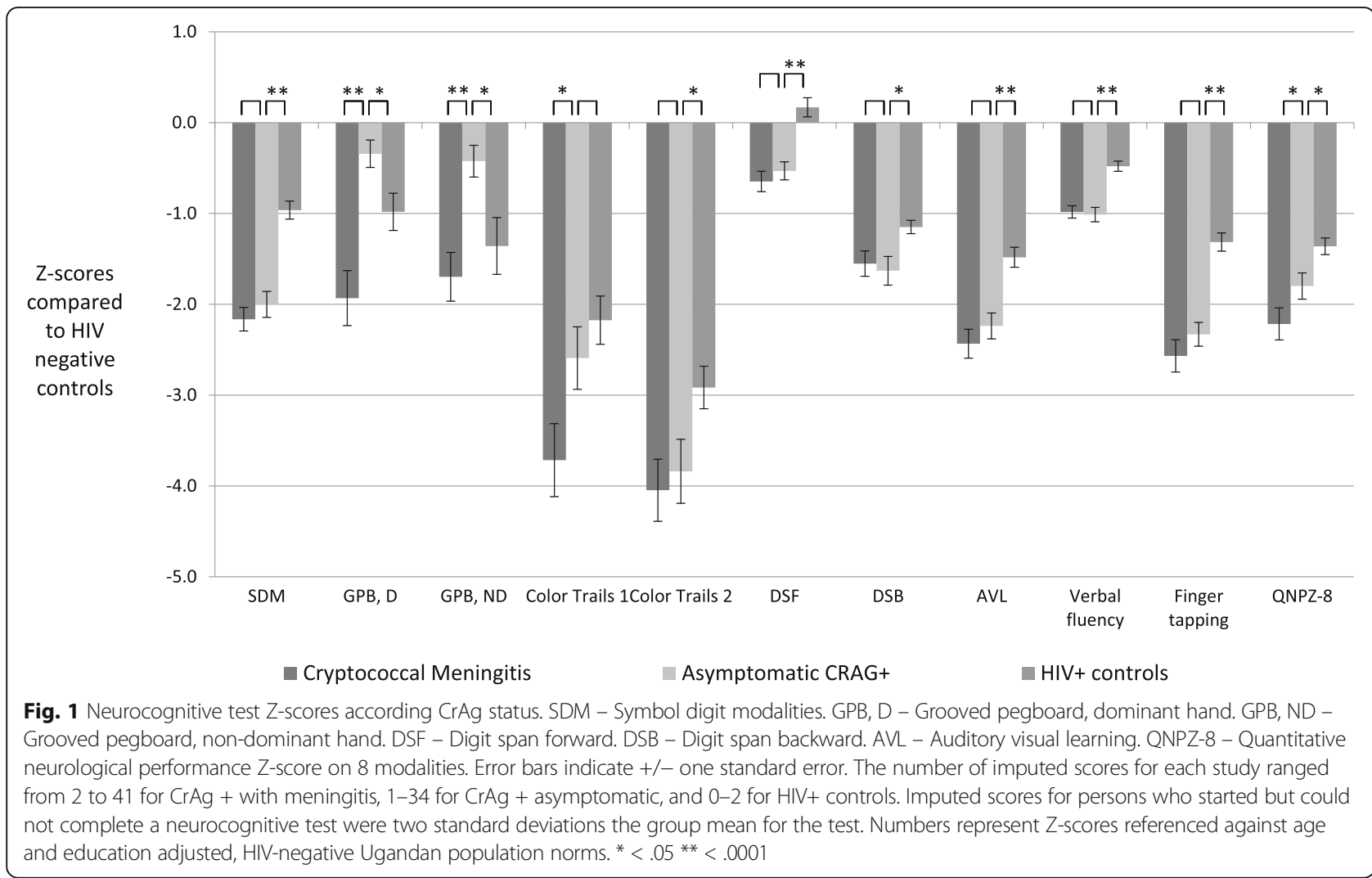

Persons with very advanced AIDS with asymptomatic cryptococcal antigenemia or recent history of cryptococcal meningitis have substantial neurocognitive dysfunction with severe deficits in executive function, verbal learning, and speed of information processing. With their neurocognitive dysfunction, this group of cryptococcal survivors and asymptomatic $\mathrm{CrAg}+$ persons likely require additional support to initially manage their ART and HIV care in order to avoid poor ART outcomes.

There are several potential explanations for the observation that asymptomatic $\mathrm{CrAg}+$ persons perform worse than HIV-infected persons without cryptococcal infection. Initial exposure to Cryptococcus is through the respiratory system, and the development of antigenemia results from dissemination from the respiratory system to the blood stream [31]. The course of disease seen thereafter is variable in time course, and asymptomatic $\mathrm{CrAg}+$ persons likely form a spectrum of infection - from those without any CNS penetration of Cryptococcus to persons with subclinical cryptococcal infection of the brain and meninges. More advanced HIV states are likely to result in increased CSF inflammation, which may increase risk of neurocognitive

Table 2 Simple and multiple linear regression analyses estimating the relationship of patient characteristics with composite neurocognitive function, QNPZ-8 score

\begin{tabular}{|c|c|c|c|c|c|c|c|}
\hline Variable & $\mathrm{N}$ & $\begin{array}{l}\text { Unadjusted univariate } \\
\text { estimate }^{a}\end{array}$ & $\begin{array}{l}95 \% \text { confidence } \\
\text { interval }\end{array}$ & $P$-value & Adjusted estimate $^{a}$ & $\begin{array}{l}95 \% \text { confidence } \\
\text { interval }\end{array}$ & $P$-value \\
\hline Study Population & 302 & & & & & & \\
\hline Cryptococcal meningitis $(N=90)$ & & -0.42 & $(-0.74,-0.09)$ & 0.01 & -0.26 & $(-0.59,0.07)$ & 0.12 \\
\hline Asymptomatic CRAG $(N=87)$ & & Referent & - & & Referent & -- & \\
\hline HIV positive controls $(N=125)$ & & 0.44 & $(0.14,0.74)$ & 0.004 & 0.42 & $(0.03,0.81)$ & 0.04 \\
\hline Age (per 10 years) & 302 & -0.20 & $(-0.37,-0.03)$ & 0.02 & -0.31 & $(-0.48,-0.15)$ & $<0.001$ \\
\hline Gender (Men as referent group) & 302 & 0.01 & $(-0.25,0.27)$ & 0.94 & -0.21 & $(-0.47,0.04)$ & 0.11 \\
\hline CD4 count (per 100 increase) & 290 & 0.16 & $(0.07,0.26)$ & 0.001 & 0.02 & $(-0.10,0.14)$ & 0.79 \\
\hline Depression $^{b}$ & 300 & -0.77 & $(-1.02,-0.53)$ & $<0.001$ & -0.56 & $(-0.82,-0.30)$ & $<0.001$ \\
\hline
\end{tabular}

${ }^{\mathrm{a}}$ Each estimate corresponds with the change in QNPZ-8 score for each incremental change in the variable

${ }^{b}$ Depression modeled as a binary variable comparing using CES-D score $>=16$ versus $<16$ as referent 
Table 3 Population characteristics and average neurocognitive test Z-scores for 73 persons with asymptomatic cryptococcal antigenemia

\begin{tabular}{|c|c|c|c|c|c|c|c|}
\hline \multirow[b]{2}{*}{ Population Characteristics } & \multicolumn{2}{|c|}{$\begin{array}{l}\text { Pre-ART visit week } 2 \\
\text { of fluconazole }\end{array}$} & \multicolumn{2}{|c|}{$\begin{array}{l}\text { Week } 4 \text { of HIV therapy week } 6 \\
\text { of fluconazole }\end{array}$} & \multicolumn{2}{|c|}{ Intra-person change } & \multirow[b]{2}{*}{$P$-value } \\
\hline & Average & SD & Average & SD & Change & SD & \\
\hline CD4 count (cells/uL), $N=60$ & 32 & \pm 30 & 80 & \pm 74 & 47 & \pm 66 & $<0.001$ \\
\hline Karnofsky score, $N=73$ & 72 & \pm 12 & 75 & \pm 11 & 2.5 & \pm 10 & 0.04 \\
\hline CES depression scale, $N=73$ & 19 & \pm 10 & 16 & \pm 10 & -3.0 & \pm 12 & 0.04 \\
\hline \multicolumn{8}{|l|}{ Neurocognitive Tests } \\
\hline Symbol digit modalities & -1.9 & \pm 1.2 & -1.5 & \pm 1.5 & 0.4 & \pm 1.0 & 0.002 \\
\hline Grooved pegboard dominant & -0.3 & \pm 1.3 & 0.1 & \pm 1.1 & 0.4 & \pm 1.4 & 0.02 \\
\hline Grooved pegboard non-dominant & -0.4 & \pm 1.5 & -0.02 & \pm 1.3 & 0.3 & \pm 1.3 & 0.03 \\
\hline Color Trails 1 & -2.4 & \pm 2.6 & -1.0 & \pm 1.7 & 1.4 & \pm 2.3 & $<0.001$ \\
\hline Color Trails 2 & -3.7 & \pm 2.6 & -1.9 & \pm 1.6 & 1.7 & \pm 2.1 & $<0.001$ \\
\hline Digit span forward & -0.5 & \pm 0.9 & -0.3 & \pm 0.9 & 0.2 & \pm 0.9 & 0.15 \\
\hline Digit span backward & -1.4 & \pm 1.4 & -1.1 & \pm 1.3 & 0.3 & \pm 1.2 & 0.03 \\
\hline Auditory verbal learning & -2.2 & \pm 1.4 & -1.2 & \pm 1.5 & 1.0 & \pm 1.2 & $<0.001$ \\
\hline Verbal fluency & -0.9 & \pm 0.7 & -0.6 & \pm 0.7 & 0.3 & \pm 0.7 & 0.005 \\
\hline Finger tapping & -2.3 & \pm 1.1 & -1.9 & \pm 1.0 & 0.4 & \pm 1.1 & 0.002 \\
\hline QNPZ-8 & -1.7 & \pm 1.0 & -1.0 & \pm 0.8 & 0.7 & \pm 0.7 & $<0.001$ \\
\hline
\end{tabular}

Numbers represent Z-scores referenced against age and education adjusted, HIV-negative Ugandan population norms. Initial testing was performed at the pre-ART visit which was 2 weeks after diagnosis of cryptococcal antigenemia and receipt of fluconazole pre-emptive therapy. Statistical testing by paired t-test

impairment or HAND [12]. Thus, the decreased cognitive function in asymptomatic CrAg + persons could be explained, at least in part, by increased prevalence of HAND.

One could similarly postulate that asymptomatic $\mathrm{CrAg}+$ persons perform poorly on neurocognitive testing because they have more advanced HIV with lower CD4 counts and functional performance. After adjusting for CD4 count, the $\mathrm{CrAg}+$ cohort effect remained while the effect of CD4 disappeared. This is most likely the result of "aggregation bias" wherein the cohort is too intricately related to the CD4 count to make a valid comparison. Unfortunately, there was an insufficient number of participants in the HIV+ control group with CD4 < $100(N=15)$ to perform an adequate subset analysis. Ideally, future studies should be conducted comparing asymptomatic $\mathrm{CrAg}+$ persons to CrAg-negative persons with similar CD4 counts. CSF sampling was not performed in the asymptomatic cohort. Some asymptomatic persons would have had detectable CSF CrAg and others would have had Cryptococcus yeasts in the brain parenchyma in the absence of CSF CrAg detectability. However, the fundamental conclusion remains that cryptococcal antigenemia in persons with low CD4 counts, even if not a causal factor for neurocognitive impairment, can be a useful marker for identifying persons with decreased neurocognitive function. This has very important implications when enrolling these persons into HIV treatment and care as there is likely a need for increased adherence support for these persons compared to the typical HIV-infected person initiating HIV care.
The second finding of the study demonstrated that asymptomatic $\mathrm{CrAg}+$ persons improved on most neurocognitive measures over a period of 4 weeks. In our prior longitudinal follow-up of persons with cryptococcal meningitis, neurocognitive performance came to within one standard deviation of HIV-negative population norms (QNPZ-8 $=-1.0 \pm 1.1$ ) and similar to HIV-infected controls (QNPZ-8 = -0.7 \pm 1.0 ) after 12 months [14]. Thus, we would expect the majority of $\mathrm{CrAg}+$ persons to normalize their neurocognitive function over time on ART [32, 33], and any additional adherence and/or social support required is likely of short duration.

One additional contributing factor that should be considered is practice effect, especially given the one-month interval between evaluations. Practice effect has been identified in previous cohorts of $\mathrm{HIV}$-infected persons undergoing neurocognitive testing $[17,34]$. Comparing longitudinal changes in neurocognitive performance in both the study and the control populations would be beneficial. Unfortunately, repeat testing of neurocognitive function in our HIV-infected control population was not performed. Aside from practice effect, it is important to note that participants demonstrated improved Karnofsky performance status over the first month of ART $(P=0.04)$. The improvement in neurocognitive function is therefore potentially explained by ART initiation with improved control of HIV infection and/or the use of pre-emptive fluconazole therapy.

There are several limitations in this study that should be considered. HIV viral load was not measured in either plasma or CSF, which may act as a potential confounder for worse neurocognitive function, although plasma levels 
would be expected to be high [35]. In the prior cryptococcal meningitis cohort, the pre-ART plasma HIV viral load was not associated with neurocognitive dysfunction [14]. Secondly, the repeat testing of neurocognitive function which showed improvement after 1 month of ART may have a component of improvement due to a practice effect, but overall appears quite promising for longer term function. As shown previously among cryptococcal meningitis survivors, their neurocognitive function normalizes to within one standard deviation of population norms within 1 year of ART [14]. Additionally, although meningitis was excluded in the HIV-infected control population, this population was not tested for CrAg. Based on a median CD4 of 233 cells/ $\mu \mathrm{L}$, there may have been approximately 3 of 100 controls who could have tested positive, and in sensitivity analyses, this was not enough to account for the findings [8].

\section{Conclusions}

In summary, asymptomatic CrAg + persons have worse neurocognitive function compared with their HIVinfected peers without CNS infection, although the exact etiology of poor neurocognitive function is unclear. There are a multitude of reasons for starting pre-emptive fluconazole treatment in HIV-infected $\mathrm{CrAg}+$ persons in the absence of meningitis. Screening is cost effective, and pre-emptive treatment with fluconazole can prevent cryptococcal meningitis and reduce early ART mortality [36]. Additional support for ART and fluconazole adherence during the first month of therapy has been associated with improved mortality [37]. As more countries roll out $\mathrm{CrAg}$ screening in populations with advanced HIV, some initial adherence support may be necessary due to the neurocognitive dysfunction present in $\mathrm{CrAg}+$ persons.

\section{Additional files}

Additional file 1: Table S1. Neuropsychological test battery and neurocognitive domains evaluated. (PDF $330 \mathrm{~kb}$ )

Additional file 2: Neurocognitive outcomes in three HIV study populations. This file contains the data generated and analyzed in this publication. (CSV $107 \mathrm{~kb})$

Additional file 3: Table S2. Neurocognitive test average Z-scores for CrAg- vs. asymptomatic CrAg + vs. cryptococcal meningitis survivors. (PDF $268 \mathrm{~kb}$ )

\footnotetext{
Abbreviations

ART: Antiretroviral therapy; AVL: Auditory visual learning; CES-D: Center for Epidemiologic Studies Depression; Cl: Confidence interval; CNS: Central nervous system; CrAg: Cryptococcal antigen; CSF: Cerebrospinal fluid; DSB: Digit span backward; DSF: Digit span forward; GPB, D: Grooved pegboard, dominant hand; GPB, ND: Grooved pegboard, non-dominant hand; HAND: HIV-associated neurocognitive disorder; IHDS: International HIV Dementia Scale; IQR: Interquartile range; QNPZ-8: Quantitative neurocognitive performance z-score; SDM: Symbol digit modalities; UCLA: University of California, Los Angeles; WHO: World Health Organization
}

\section{Acknowledgements}

We are grateful for the medical care of the participants by Drs. Abdu Musubire and Henry Nabeta (Infectious Disease Institute, Makerere University). We thank the research assistants: Alice Namudde and Charles Ssebunya (Infectious Disease Institute, Makerere University) for the neurocognitive data collection. We thank Dr. Ned Sacktor (Johns Hopkins Hospital) for his pioneering work on neurocognitive function in Africa to enable comparisons. We dedicate this manuscript to the memory of Dr. Kate Birkenkamp

The conclusions, findings, and opinions expressed by authors contributing to this journal do not necessarily reflect the official position of the U.S.

Department of Health and Human Services, the Public Health Service, the Centers for Disease Control and Prevention, or the authors' affiliated institutions.

\section{Funding}

This research was made possible through support from the Centers for Disease Control and Prevention (U01GH000517), Fogarty International Center and National Institute of Neurologic Diseases and Stroke (R21NS065713, R01NS086312, R25TW009345), and National Institute of Allergy and Infectious Diseases (U01Al089244, T32Al055433, K24Al096925). These funding bodies had no role in the study design, in the collection, analysis, or interpretation of data, or in the writing of the manuscript.

Availability of data and materials

All data generated and analyzed during this study are included in this published article as a supplemental file (asxcrag.csv).

\section{Authors' contributions}

MPM: Statistical analysis and interpretation, drafting of the manuscript. NN: Study design, data interpretation, editing of manuscript. BMM: Data collection, interpretation, and analysis, editing of manuscript. RR: Study design, editing of manuscript. JR: Study design, study implementation, editing of manuscript. EN: Study design, study implementation, data coordination. DAW: Study design, study implementation. KHH: Study design, data analysis and interpretation. AK: Study design, editing of manuscript. MAR: Study design, editing of manuscript. RDC: Study design, editing of manuscript. NCB: Study design and implementation, editing of manuscript. KB: Study design, data collection. YCM: Study design, editing of manuscript. PRB: Study design, editing of manuscript. JEK: Data interpretation, editing of manuscript. AK: Study design, editing of manuscript. DBM: Study design, data interpretation, editing of manuscript. DRB: Study design, data interpretation, editing of manuscript. All authors read and approved the final manuscript.

\section{Competing interests}

The authors declare that they have no competing interests.

\section{Consent for publication}

Not applicable.

\section{Ethics approval and consent to participate}

Ethics approval was obtained for all three cohorts. Name and committee references numbers where applicable are indicated below. Cohort A, Cryptococcal meningitis cohort: National HIV/AIDS Research Committee-ARC 113. Uganda National Council for Science and Technology HS900. Infectious Disease Institute, Scientific Review Committee. Cohort B, Asymptomatic CrAg +: Ugandan National Council for Science and Technology HS 1254. Joint Clinical Research Centre IRB. Infectious Disease Institute, Scientific Review Committee. University of Minnesota IRB: 1202 M10701. Uganda National Drug Authority 3.95/ESR/NDA/D1D:I2/2012. Johns Hopkins University IRB: NA_00078248. Cohort C, HIV+ controls: Faculty of Medicine Ethics and Research Committee, Makerere Medical School. Uganda National Council of Science and Technology.

\section{Publisher's Note}

Springer Nature remains neutral with regard to jurisdictional claims in published maps and institutional affiliations. 


\section{Author details}

'Division of Infectious Diseases and International Medicine, Department of Medicine, University of Minnesota, 3-222 MTRF, 2001 6th St SE, Minneapolis, MN 55455, USA. ${ }^{2}$ Infectious Disease Institute, Makerere University, Kampala, Uganda. ${ }^{3}$ Department of Medicine, School of Medicine, Makerere University College of Health Sciences, Kampala, Uganda. ${ }^{4}$ Department of Psychiatry, School of Medicine, Makerere University College of Health Sciences, Kampala, Uganda. ${ }^{5} \mathrm{~S} c h o o l$ of Public Health, University of Minnesota, Minneapolis, MN, USA. ${ }^{6}$ Johns Hopkins School of Medicine, Baltimore, MD, USA. "Division of Global HIV and TB, Center for Global Health, Centers for Disease Control and Prevention, Atlanta, GA, USA. ${ }^{8}$ Infectious Disease Institute, Mulago Hospital Complex, Kampala, Uganda.

\section{Received: 12 November 2016 Accepted: 9 May 2017} Published online: 12 June 2017

\section{References}

1. Meyer AC, Kendi CK, Penner JA, Odhiambo N, Otieno B, Omondi E, et al. The impact of routine cryptococcal antigen screening on survival among HIV-infected individuals with advanced immunosuppression in Kenya. Trop. Med. Int. Heal. 2013;18:495-503.

2. French N, Gray K, Watera C, Nakiyingi J, Lugada E, Moore M, et al. Cryptococcal infection in a cohort of HIV-1-infected Ugandan adults. AIDS 2002;16:1031-8

3. Park BJ, Wannemuehler KA, Marston BJ, Govender N, Pappas PG, Chiller TM. Estimation of the current global burden of cryptococcal meningitis among persons living with HIV/AIDS. AIDS. 2009;23:525-30.

4. Castelblanco RL, Lee M, Hasbun R. Epidemiology of bacterial meningitis in the USA from 1997 to 2010: a population-based observational study. Lancet Infect Dis. 2014;14:813-9. [cited 2016 Jan 5]. Available from: http://www. ncbi.nlm.nih.gov/pubmed/25104307.

5. Pyrgos V, Seitz AE, Steiner CA, Prevots DR, Williamson PR. Epidemiology of cryptococcal meningitis in the US: 1997-2009. PLoS One. 2013;8:e56269. [cited 2015 Dec 9]. Available from: http://www.pubmedcentral.nih.gov/ articlerender.fcgi?artid $=3574138 \&$ tool $=$ pmcentrez\&rendertype $=$ abstract.

6. Liechty CA, Solberg P, Were W, Ekwaru JP, Ransom RL, Weidle PJ, et al. Asymptomatic serum cryptococcal antigenemia and early mortality during antiretroviral therapy in rural Uganda. Trop Med Int Heal. 2007;12:929-35.

7. Ganiem AR, Indrati AR, Wisaksana R, Meijerink H, Van Der Ven A, Alisjahbana B, et al. Asymptomatic cryptococcal antigenemia is associated with mortality among HIV-positive patients in Indonesia. J Int AIDS Soc. 2014;17:1-7.

8. Meya DB, Manabe YC, Castelnuovo B, Cook BA, Elbireer AM, Kambugu A, et al. Cost-effectiveness of serum cryptococcal antigen screening to prevent deaths among HIV-infected persons with a CD4+ cell count $<$ or $=100$ cells/microL who start HIV therapy in resource-limited settings. Clin Infect Dis. 2010;51:448-55.

9. Jarvis JN, Lawn SD, Vogt M, Bangani N, Wood R, Harrison TS. Screening for cryptococcal antigenemia in patients accessing an antiretroviral treatment program in South Africa. Clin Infect Dis. 2009:48:856-62.

10. World Health Organization. Rapid advice: diagnosis, prevention and management of cryptococcal disease in HIV-infected adults, adolescents and children. Geneva World Heal Organ. 2011. [cited 2015 Feb 6]. Available from: http://whqlibdoc.who.int/publications/2011/9789241502979_eng.pdf.

11. Masur H, Brooks JT, Benson CA, Holmes KK, Pau AK, Kaplan JE. Prevention and treatment of opportunistic infections in HIV-infected adults and adolescents: updated guidelines from the Centers for Disease Control and Prevention, National Institutes of Health, and HIV Medicine Association of the Infectious Diseases soc. Clin Infect Dis. 2014;58:1308-11. [cited 2016 Jan 5]. Available from: http://www.pubmedcentral.nih.gov/articlerender. fcgi?artid=3982842\&tool=pmcentrez\&rendertype=abstract.

12. Zayyad Z, Spudich S. Neuropathogenesis of HIV: from initial Neuroinvasion to HIV-associated Neurocognitive disorder (HAND). Curr HIV/AIDS Rep. 2015;12: 16-24. Available from: http://link.springer.com/10.1007/s11904-014-0255-3.

13. Wong MH, Robertson K, Nakasuija N, Skolasky R, Musisi S, Katabira E, et al. Frequency of and risk factors for HIV dementia in an HIV clinic in subSaharan Africa. Neurology. 2007;68:350-5. [cited 2017 Apr 16]. Available from: http://www.ncbi.nlm.nih.gov/pubmed/17261681.

14. Carlson RD, Rolfes MA, Birkenkamp KE, Nakasujia N, Rajasingham R, Meya $D B$, et al. Predictors of neurocognitive outcomes on antiretroviral therapy after cryptococcal meningitis: a prospective cohort study. Metab Brain Dis. 2014;29:269-79.
15. Levine AJ, Hinkin CH, Ando K, Santangelo G, Martinez M, Valdes-Sueiras M, et al. An exploratory study of long-term neurocognitive outcomes following recovery from opportunistic brain infections in HIV+ adults. J Clin Exp Neuropsychol NIH Public Access. 2008;30:836-43. [cited 2017 Apr 16]. Available from: http://www.ncbi.nlm.nih.gov/pubmed/18608693.

16. Boulware DR, Meya DB, Muzoora C, Rolfes MA, Huppler Hullsiek K, Musubire A, et al. Timing of antiretroviral therapy after diagnosis of cryptococcal meningitis. N Engl J Med. 2014;370:2487-98. Available from: http://www. ncbi.n/m.nih.gov/pubmed/24963568.

17. Sacktor N, Nakajujja N, Okonkwo O, Skolasky RL, Robertson K, Musisi S, et al. Longitudinal neuropsychological test performance among HIV seropositive individuals in Uganda. J Neuro-Oncol. 2013;19:48-56.

18. Wechsler D. Wechsler adult intelligence scale - revised (WAIS-R). San Antonio, TX: Psychological Corporation; 1981.

19. D'Elia LF, Satz P, Uchiyama CL, White T. Color Trails test. Professional manual. Psychological Assessment Resources: Odessa, FL; 1996.

20. Maj M, D'Elia L, Satz P, Janssen R, Zaudig M, Uchiyama C, et al. Evaluation of two new neuropsychological tests designed to minimize cultural bias in the assessment of HIV-1 seropositive persons: a WHO study. Arch Clin Neuropsychol. 1993;8:123-35.

21. Spreen O, Strauss E. A compendium of neuropsychological tests: administration, norms, and commentary. 2nd ed. New York: Oxford University Press; 1998

22. Reitan RM. Manual for administration of neuropsychological test batteries for adults and children. Bloomington, IN: Indiana University Press; 1969.

23. Klove H. Clinical Neuropsychology. Med Clin North Am. 1963;47:1647-58. [cited 2015 Feb 23]. Available from: http://www.ncbi.nlm.nih.gov/pubmed/ 14078168

24. Sacktor NC, Wong M, Nakasujja N, Skolasky RL, Selnes OA, Musisi S, et al. The International HIV dementia scale: a new rapid screening test for HIV dementia. AIDS. 2005;19:1367-74.

25. Robertson KR, Nakasujia N, Wong M, Musisi S, Katabira E, Parsons TD, et al. Pattern of neuropsychological performance among HIV positive patients in Uganda. BMC Neurol. 2007;7:8.

26. Price RW, Yiannoutsos CT, Clifford DB, Zaborski L, Tselis A, Sidtis JJ, et al. Neurological outcomes in late HIV infection: adverse impact of neurological impairment on survival and protective effect of antiviral therapy. AIDS clinical trial group and neurological AIDS research consortium study team. AIDS. 1999;13:1677-85.

27. Karnofsky DA, Burchenal JH. The clinical evaluation of chemotherapeutic agents in cancer. In: Macleod CM, editor. Eval. Chemother. Agents. New York: Columbia University Press; 1949. p. 191-205.

28. Radloff LS. The CES-D scale: a self-reported depression scale for research in the general population. Appl Psychol Meas. 1977;1:385-401.

29. Beekman ATF, Deeg DJH, van Limbeek J, Braam AW, de Vries MZ, van Tilburg W. Criterion validity of the Center for Epidemiologic Studies Depression scale (CES-D): results from a community-based sample of older subject in the Netherlands. Psychol Med. 1997;27:231-5. Available from: http://dare.ubvu.vu.nl/handle/1871/23024.

30. Lewinsohn PM, Seeley JR, Roberts RE, Allen NB. Center for Epidemiologic studies depression scale (CES-D) as a screening instrument for depression among community-residing older adults. Psychol Aging. 1997;12:277-87. cited 2016 Nov 3]. Available from: http://www.ncbi.nlm.nih.gov/pubmed/ 9189988.

31. Feldmesser M, Harris C, Reichberg S, Khan S, Casadevall A. Serum cryptococcal antigen in patients with AIDS. Clin Infect Dis. 1996;23:827-30.

32. Robertson KR, Robertson WT, Ford S, Watson D, Fiscus S, Harp AG, et al. Highly active antiretroviral therapy improves neurocognitive functioning. J Acquir Immune Defic Syndr. 2004;36:562-6.

33. Sacktor N, Nakasujja N, Skolasky R, Robertson K, Wong M, Musisi S, et al. Antiretroviral therapy improves cognitive impairment in HIV+ individuals in sub-Saharan Africa. Neurology. 2006;67:311-4.

34. Grund B, Wright EJ, Brew BJ, Price RW, Roediger MP, Bain MP, et al. Improved neurocognitive test performance in both arms of the SMART study: impact of practice effect. J Neuro-Oncol. 2013;19:383-92.

35. Butler EK, Boulware DR, Bohjanen PR, Meya DB. Long term 5-year survival of persons with Cryptococcal meningitis or asymptomatic subclinical Antigenemia in Uganda. PLoS One. 2012;7:5-9.

36. Rajasingham R, Meya DB, Boulware DR. Integrating Cryptococcal antigen screening and pre-emptive treatment into routine HIV care. J Acquir Immune Defic Syndr. 2012;59:e85-91. [cited 2015 Feb 23]. Available from: 
http://www.pubmedcentral.nih.gov/articlerender.fcgi?artid=3311156\&tool= pmcentrez\&rendertype $=$ abstract.

37. Mfinanga S, Chanda D, Kivuyo SL, Guinness L, Bottomley C, Simms V, et al. Cryptococcal meningitis screening and community-based early adherence support in people with advanced HIV infection starting antiretroviral therapy in Tanzania and Zambia: an open-label, randomised controlled trial. Lancet (London, England). 2015;385:2173-2182. [cited 2015 Aug 13]. Available from: http://www.sciencedirect.com/science/article/pii/ S0140673615601647.

Submit your next manuscript to BioMed Central and we will help you at every step:

- We accept pre-submission inquiries

- Our selector tool helps you to find the most relevant journal

- We provide round the clock customer support

- Convenient online submission

- Thorough peer review

- Inclusion in PubMed and all major indexing services

- Maximum visibility for your research

Submit your manuscript at www.biomedcentral.com/submit
Biomed Central 